\title{
Correlation of Resistin levels with risk factors in type 2 diabetes mellitus patients with ischemic stroke
}

\author{
TRameswari ${ }^{1}, \mathrm{G}$ Ezhil $^{2 *}$ \\ 1,2Assistant Professor, Department of Biochemistry, Coimbatore medical college, Coimbatore, Tamil Nadu-641014, INDIA. \\ Email:ezhil2085@gmail.com
}

Abstract Background: Ischemic stroke in diabetic individuals is complex in nature with contributions from both environmental and genetic factors. Resistin links the metabolic dysregulation to atherosclerosis through selective inhibition of PKB insulin signalling and activation of MAPK pathway. Aim: To find the correlation of resistin levels with risk factors in type 2 diabetes mellitus patients with ischemic stroke. Material and Methods: Study population was grouped as Group 1: 60 unrelated Type 2 diabetic adults diagnosed with ischemic stroke and Group 2: 60 unrelated Type 2 diabetic adults without ischemic stroke and controls including 60 individuals not fitted in either group.Results: There was a significant weak positive correlation between serum resistin levels and age, waist circumference, fasting plasma glucose, lipid profile except for HDL-c levels. Significant weak negative correlation was found between serum resistin levels and HDLc. Conclusion: Among patients with diabetes several risk factors play a role together to promote the development of ischemic stroke. Significant correlation found between serum resistin levels and age, waist circumference, fasting plasma glucose, lipid profile except for HDL-c levels.

Key Word: Type 2 diabetes mellitus, ischemic stroke, resistin level, risk factors

*Address for Correspondence:

Dr G Ezhil, Assistant Professor, Department of Biochemistry, Coimbatore medical college, Coimbatore, Tamil Nadu-641014, INDIA.

Email:ezhil2085@gmail.com

Received Date: 26/01/2019 Revised Date: 20/02/2019 Accepted Date: 05/03/2019

DOI: https://doi.org/10.26611/1002931

\begin{tabular}{|l|l|}
\hline \multicolumn{2}{|c|}{ Access this article online } \\
\hline Quick Response Code: & Website: \\
\hline & www.medpulse.in \\
\cline { 2 - 2 } & \\
\hline
\end{tabular}

\section{INTRODUCTION}

Type 2 Diabetes mellitus affects large number of people worldwide. It is related with cerebrovascular and cardiovascular complications. Diabetes is continuing as global epidemic and it is particularly concentrated in Indian subcontinent ${ }^{1}$ and that there is also a greater propensity of Indian population developingstroke. ${ }^{2} \mathrm{~A}$ cerebrovascular complication that is more commonly associated with diabetes is ischemic stroke. ${ }^{3}$ Diabetes is an independent risk factor for ischemic stroke. Diabetes mellitusis associated with 2 to 5 fold increased risk for the first or recurrent ischemic stroke. ${ }^{4,5}$ Diabetes as an independent factor affects the stroke in age of onset, in its types, also in prognosis. In India, it has been found that $80 \%$ of stroke are due to ischemia. ${ }^{6}$ Identifying the factors that predisposes the diabetic patients to macroangiopathy will be helpful in monitoring the disease progression. Ischemic stroke in diabetic individuals is complex in nature with contributions from both environmental and genetic factors. Ischemic stroke is a complicated vascular disease and it is affected by many factors. Conventional risk factors can explain only half of the ischemic stroke incidence. ${ }^{7}$ Resistin, is a11.4 $\mathrm{kDa}$ protein rich in cysteine residues. This is expressed mainly in monocytes and macrophages. Though it was discovered from the adipose tissue, later it is reported to be secreted from macrophages infiltrating the adipose tissue and preadipocytes. It is involved in inflammatory and metabolic signals. ${ }^{8}$ Thus resistin could be a link between diabetes and ischemic stroke. Resistin links the metabolic dysregulation to atherosclerosis through selective inhibition ofPKB insulin signalling and 
activation of MAPK pathway. The gene, RETN at chromosome 19 p13.2 codes for human resistin. ${ }^{9}$ Thus, this study was conducted with an aim to find the correlation of resistin levels with risk factors in type 2 diabetes mellitus patients with ischemic stroke.

\section{MATERIAL AND METHODS}

This case control study was approved by ethical committee. All the participating individuals and their relatives were informed about the study.

Inclusion criteria: The study population was categorised into two groups:

Group 1: included 60 unrelated Type 2 diabetic adults diagnosed with ischemic stroke of age around 40-70 years (32 males and 28 females). This study population was selected among the atherothrombotic stroke patients admitted in the neurology and medicine wards.

Group 2: Included 60 unrelated Type 2 diabetic adults without ischemic stroke of age around 40-70 years (33 males and 27 females). These individuals were selected from diabetology outpatient department. They were diagnosed diabetic patients without any history and symptoms of stroke.

Exclusion criteria: Type 2 diabetics with other chronic illness such as renal or hepatic failure, autoimmune diseases, neoplasms, coagulopathies, myocardial infarction, family history of autoimmune or chronic inflammatory diseases and those on thiazolidones group of hypoglycemicdrugs were excluded from both the groups 1 and 2. Diabetics with less than 5 years of duration of diabetes were also excluded from both the goups. Ischemic stroke patients with known embolic source were excluded from group 2 .

Group 3 (Control): 60 individuals of age around 40-60 years (29 males and 31 females) were selected as controls. Exclusion criteria same as above is applied to control group. In addition, they should not be diabetics and there should not be history of stroke. Age, sex and other confounding factors like smoking and alcoholism were matched. The definition of stroke was set as neurological deficit of abrupt onset due to vascular originlasting longer than 24 hours. Ischemic stroke patients with history of diabetes were diagnosed with clinical history, neurological examination and brain computer tomography (CT) scan to rule out hemorrhagic stroke and then included in the study. This was followed by echocardiography and carotid doppler to rule out the embolic source.

\section{Characteristics of study population}

- Participant's baseline information and anthropometric measurements were recorded.

- Assessment of vascular risk factors were also done.
In this study some of the risk factors were defined as:

- Hypertension defined as previous diagnosis of hypertension by physician and/or treatment with antihypertensive drugs.

- A positive smoking and alcohol consumption history were recorded if the individuals had smoked or consumed alcohol regularly at any time in their lives respectively.

Sample collection: Blood was collected after an overnight fasting of 8-12 hours. About $4 \mathrm{ml}$ of blood was collected from the antecubital vein of the subjects by aseptic procedures. $2 \mathrm{~mL}$ was transferred into a EDTA tubes and another $2 \mathrm{~mL}$ was transferred in to plain non additive tubes. Blood was collected from the stroke patients after 7-10days of the onset of stroke to avoid the transient early changes of biochemical markersin the blood. The plain non additive tubes were allowed to clotfor 30 minutes and then centrifuged for 15 minutes at $3000 \mathrm{rpm}$. Serum was separated, collected in $2 \mathrm{ml}$ microcentrifuge tubes and stored at $-80 \mathrm{c}$ for the estimation of resistin.

Estimation of serum resistin: Serum resistin was measured by Sandwich Enzyme Linked Immunosorbent Assay by using Quantikine- R and D Systems. This assay reagent is highly specific detects only recombinant and human resistin. No significant cross reactivity was observed with analytes such as leptin and RELM $\beta$. The intra assay precision is $4.7 \%$ and the inter assay precision is $8.4 \%$.

Resistin standard preparation: All the reagents were in room temperature. $1 \mathrm{~mL}$ of distilled water is added to reconstitute the resistin standard. The stock solution of resistin standard of $100 \mathrm{ng} / \mathrm{mL}$ was prepared. The following serial dilution of stock standard solution(100ng/mL) was done with the calibrator diluent.

Sample preparation: Serum samples were brought to room temperature. 5-fold dilution of sample is done as $50 \mu \mathrm{L}$ of sample $+200 \mu \mathrm{L}$ of calibrator diluent.

Assay procedure: $100 \mu \mathrm{L}$ of assay diluent was added to each well of ELISA microplate. Then $100 \mu \mathrm{L}$ of resistin standards S1 to S8 were added to the wells. $100 \mu \mathrm{L}$ of samples were added into the remaining wells. The plate was covered with adhesive strips and then incubated for 2 hours at room temperature. Standards and samples added were recorded in the plate layout. In the meantime $20 \mathrm{~mL}$ of wash buffer concentrate was diluted to $500 \mathrm{~mL}$ by adding distilled water. The program was set in the automated ELIS Aplate washer with soaking time as 30 seconds and repeating the wash three times.

After 2 hours of incubation, the ELISA microplate was placed in the ELISA washer. After the last wash, the plate was inverted and blotted against the clean paper to remove the remaining wash buffer. $200 \mu \mathrm{L}$ of resistin 
conjugate was then added to each well. The plate was covered with adhesive strip and incubated for 2 hours at room temperature. The plate was washed with the same program in the automated ELISA washer. Substrate solution was prepared by mixing the Colour Reagent A and $\mathrm{B}$ and protected from light by covering with aluminium foil. $200 \mu \mathrm{L}$ of Substrate solution is added to each well. The plate is then incubated for 30 minutes and covered with adhesive strip to protect from light.. $50 \mu \mathrm{L}$ of Stop solution was added to each well. On adding the Stop solution, blue colour in the wells changed to yellow colour. The program was set in the ELISA microplate reader to determine the optical density of each well at $450 \mathrm{~nm}$.

Statistical analysis: Data were analysed using SPSS statistical software version 16 and was considered as statistically significant if the $\mathrm{p}$ value is less than 0.05 . Age, Waist circumference, fasting plasma glucose and fasting lipid profile were compared between the three study groups by ANOVA. Gender, smoking, alcoholism hypertension were compared between the three study groups by chi-square test. Duration of diabetes, fasting plasma glucose, hypertension and fasting lipid profile were compared between the two study groups by students' independent t-test. Correlation of the parameters namely age, waist circumference, fasting plasma glucose and fasting lipid profile with serum resistin levels were found out by Pearson's correlation analysis.

\section{RESULTS}

There were no significant difference in the distribution of age and sex among the study subjects, showing that this is an age and sex matched case-control study. Also there were no significant difference between the study groups in smoking, alcoholism and hypertension. This shows that these risk factors were also matched among the three groups of this study.We obtained a significant difference between the study groups in waist circumference, concentration of fasting plasma glucose, triglycerides, HDL-cholesterol, TC/HDL-c ratio and NonHDLcholesterol with the $\mathrm{p}$ value $<0.001$ But there is no significant difference in the concentration of total cholesterol and LDL-cholesterol between study groups. Serum resistin levels were also significantly high in type 2 DM patients with ischemic stroke $(39.5 \pm 21.2 \mathrm{ng} / \mathrm{mL})$ compared to type $2 \mathrm{DM}$ patients without ischemic stroke $(25.9 \pm 13.6 \mathrm{ng} / \mathrm{mL})$ and controls $(21 \pm 9.58 \mathrm{ng} / \mathrm{mL})$ with $\mathrm{p}$ value $<0.001$.

\begin{tabular}{|c|c|c|c|c|}
\hline Parameters & Controls & $\begin{array}{l}\text { Diabetics without } \\
\text { ischemic stroke }\end{array}$ & $\begin{array}{l}\text { Diabetics with } \\
\text { ischemic stroke }\end{array}$ & $\mathrm{p}$ value \\
\hline Age & $55.5 \pm 5.02$ & $55.7 \pm 8.66$ & $57.3 \pm 6.52$ & 0.278 \\
\hline Sex male & $29(48.3 \%)$ & $32(53.3 \%)$ & $33(55 \%)$ & \\
\hline Female & $31(51.6 \%)$ & $28(46.6 \%)$ & $27(45 \%)$ & 0.749 \\
\hline Smoking & $12(20 \%)$ & $12(20 \%)$ & $18(30 \%)$ & 0.327 \\
\hline Alcohol & $17(28.3 \%)$ & $14(23.3 \%)$ & $13(21.7 \%)$ & 0.676 \\
\hline Hypertension & $14(23.3 \%)$ & $15(25 \%)$ & $18(30 \%)$ & 0.688 \\
\hline Waist cricumference & $85.5 \pm 6.5$ & $88.6 \pm 5.14$ & $90.7 \pm 5.6$ & $\varangle .001 * *$ \\
\hline $\mathrm{FBS}(\mathrm{mg} / \mathrm{dL})$ & $91.05 \pm 8.3$ & $166.7 \pm 42.1$ & $182.72 \pm 57.3$ & $\varangle .001^{* *}$ \\
\hline Total cholesterol (mg/dL) & $161.6 \pm 23.2$ & $162.7 \pm 27.2$ & $170.7 \pm 22.73$ & 0.084 \\
\hline Triglycerides $(\mathrm{mg} / \mathrm{dL})$ & $111.07 \pm 20.6$ & $135.3 \pm 51.16$ & $158.2 \pm 45.72$ & $\varangle 0.001 * *$ \\
\hline LDL-c $(\mathrm{mg} / \mathrm{dL})$ & $92.8 \pm 22.3$ & $91.07 \pm 29.19$ & $97.19 \pm 24.2$ & 0.399 \\
\hline $\mathrm{HDL}-\mathrm{c}(\mathrm{mg} / \mathrm{dL})$ & $46.6 \pm 6.34$ & $44.24 \pm 6.4$ & $41.9 \pm 5.55$ & $\varangle .001 * *$ \\
\hline TC/HDL-cratio & $3.52 \pm 0.701$ & $3.767 \pm 0.937$ & $4.13 \pm 0.72$ & $\varangle 0.001^{* *}$ \\
\hline Non HDL-c (mg/dL) & $115 \pm 23.8$ & $118.15 \pm 30.15$ & $128.8 \pm 22.89$ & $0.01 * *$ \\
\hline Resistin (ng/mL) & $21 \pm 9.58$ & $25.99 \pm 13.69$ & $39.5 \pm 21.2$ & $\varangle .001^{* *}$ \\
\hline
\end{tabular}

* highly significant at $\mathrm{p}<0.001$

Table 2 shows the comparison of characteristics between type 2 DM patients without ischemic stroke and type 2 DM patients with ischemic stroke. Significant differences were found between these two groups only in waist circumference, levels of triglycerides, HDL-c, Non HDL$\mathrm{c}$ and TC/HDL-c ratio. Other parameters namely age, sex, smoking, alcoholism, hypertension, duration of diabetes, levels of fasting glucose, total cholesterol and LDL-c were found to be statistically indifferent and hence these confounding factors were similar in type 2 DM patients with or without ischemic stroke. But serum resistin levels were significantly increased in type $2 \mathrm{DM}$ patients with ischemic stroke(39.5 $\pm 21.2 \mathrm{ng} / \mathrm{mL})$ compared to type 2 DM patients without ischemic stroke $(25.9 \pm 13.6 \mathrm{ng} / \mathrm{mL})$ with $\mathrm{p}$ value of $<0.01$. 
Table 2: comparisons of characteristics between diabetics Without ischemic stroke and diabetics with ischemic stroke

\begin{tabular}{|c|c|c|c|}
\hline Parameters & $\begin{array}{l}\text { Diabetics without } \\
\text { Ischemic stroke }\end{array}$ & $\begin{array}{l}\text { Diabetics with } \\
\text { Ischemic stroke }\end{array}$ & $p$ value \\
\hline Age & $55.7 \pm 8.66$ & $57.3 \pm 6.52$ & 0.09 \\
\hline Sexmale & $32(53.3 \%)$ & $33(55 \%)$ & \\
\hline Female & $28(46.6 \%)$ & $27(45 \%)$ & 0.855 \\
\hline Smoking & $12(20 \%)$ & $18(30 \%)$ & 0.29 \\
\hline Alcohol & $14(23.3 \%)$ & $13(21.7 \%)$ & 1.00 \\
\hline Hypertension & $15(25 \%)$ & $18(30 \%)$ & 0.54 \\
\hline Duration of diabetes (years) & $7.06 \pm 4.3$ & $6.56 \pm 3.3$ & 0.481 \\
\hline Waist circumference & $88.6 \pm 5.4$ & $90.7 \pm 5.6$ & $0.03 *$ \\
\hline $\mathrm{FBS}(\mathrm{mg} / \mathrm{dL})$ & $166.7 \pm 42.1$ & $182.72 \pm 57.3$ & 0.133 \\
\hline Total cholesterol (mg/dL) & $162.7 \pm 27.2$ & $170.7 \pm 22.73$ & 0.075 \\
\hline Triglycerides(mg/dL) & $135.3 \pm 51.16$ & $158.2 \pm 45.72$ & $0.011 *$ \\
\hline LDL-c (mg/dL) & $91.07 \pm 29.19$ & $97.19 \pm 24.2$ & 0.214 \\
\hline HDL-c (mg/dL) & $44.24 \pm 6.4$ & $41.9 \pm 5.55$ & $0.035 *$ \\
\hline TC/HDL-c RATIO & $3.767 \pm 0.937$ & $4.13 \pm 0.72$ & $0.017 *$ \\
\hline Non HDL-c (mg/dL) & $118.15 \pm 30.15$ & $128.8 \pm 22.89$ & $0.031 *$ \\
\hline Resistin (ng/mL) & $25.99+13.69$ & $39.5 \pm 2.12$ & $\varangle .001 * *$ \\
\hline
\end{tabular}

able 3 shows the correlation of serum resistin levels with age, waist circumference, fasting plasma glucose and fasting lipid profile of study population. There was a significant weak positive correlation between serum resistin levels and age, waist circumference, fasting plasma glucose, lipid profile except for HDL-c levels. Significant weak negative correlation was found between serum resistin levels and HDL-c.

Table 3: Correlation of resistin levels with other parameters in study groups

\begin{tabular}{|c|c|c|}
\hline Parameters & Correlation & Resistin \\
\hline \multirow{4}{*}{ Age } & Pearson Correlation & $.229^{* *}$ \\
\hline & Sig. (2-tailed) & .002 \\
\hline & $\mathrm{N}$ & 180 \\
\hline & Pearson Correlation & $.469^{* *}$ \\
\hline \multirow[t]{3}{*}{ Waist circumference } & Sig. (2-tailed) & .000 \\
\hline & $\mathrm{N}$ & 180 \\
\hline & Pearson Co rrelation & $.334^{* *}$ \\
\hline \multirow[t]{3}{*}{ Fasting glucose } & Sig. (2-tailed) & .000 \\
\hline & $\mathrm{N}$ & 180 \\
\hline & Pearson Correlation & $.152^{*}$ \\
\hline \multirow[t]{3}{*}{ Total cholesterol } & Sig. (2-tailed) & .042 \\
\hline & $\mathrm{N}$ & 180 \\
\hline & Pearson Correlation & $.298^{* *}$ \\
\hline \multirow[t]{3}{*}{ Triglycerides } & Sig. (2-tailed) & .000 \\
\hline & $\mathrm{N}$ & 180 \\
\hline & Pearson Correlation & $-.256^{* *}$ \\
\hline \multirow[t]{3}{*}{ HDL-C } & Sig. (2-tailed) & .001 \\
\hline & $\mathrm{N}$ & 180 \\
\hline & Pearson Correlation & $.271^{* *}$ \\
\hline \multirow[t]{3}{*}{ TC/HDL-c ratio } & Sig. (2-tailed) & .000 \\
\hline & $\mathrm{N}$ & 180 \\
\hline & Pearson Correlation & $.206^{* *}$ \\
\hline \multirow[t]{2}{*}{ Non HDL-C } & Sig. (2-tailed) & .006 \\
\hline & $\mathrm{N}$ & 180 \\
\hline
\end{tabular}




\section{DISCUSSION}

As diabetes is on increasing incidence, the morbidity and mortality due to diabetic vascular disease is also on parallel rise. Diabetes is found to be an independent risk factor of ischemic stroke. Diabetes mellitus affects the clinical manifestation of ischemic stroke in many aspects like age of onset, type of stroke, prognosis, recurrence and mortality. The reason for the increased risk of stroke in diabetes may be due to the molecular link between the various metabolic, hemodynamic and inflammatory signalling pathways. Resistin is plays a multirole in insulin resistance, inflammation and hence on atherosclerosis. More studies have been done on the development of cardio vascular complication in diabetes mellitus but not so in cerebro vascular disease. Diabetics with same duration of diabetes and glycemia status differs in their susceptibility to ischemic stroke. This points to the role of genetic factors in susceptibility of diabetics to ischemic stroke. Serum resistin levels were positively correlated with age, waist circumference, fasting glucose, total cholesterol, LDL-cholesterol, TC/HDL-C ratio and Non HDL-c. Resistin levels were negatively correlated with HDL-c. But all these correlations had low correlation coefficient value and this may be due to clustered distribution of these parameters in the study population. Even if there is weak correlation, it implies that serum resistin levels increases with age and waist circumference. It also shows that fasting glucose, total cholesterol, LDL-c, TC/HDL-c ratio and Non HDL-c increases with increase in serum resisitn levels. This similar to the study on Indian females where resistin levels were positively correlated with waist circumference, plasma glucose, serum triglycerides, serum cholesterol, and negatively correlated with HDLc. ${ }^{10}$ Similarly it has been reported by Osawa et al in Japanese population. ${ }^{11}$ According to Surapon et al.,2010, TC/HDL-c ratio and Non HDL-can be considered as marker of insulin resistance. ${ }^{12}$ In this study it has been shown that there was a significant association of resistin levels with HDL-c, TC/HDL-c ratio, non HDL-c and hence with insulin resistance.

\section{CONCLUSION}

Among patients with diabetes several risk factors play a role together to promote the development of ischemic stroke. In the analysis of these risk factors can be identified diabetes-specific factors such as hyperglycemia and vascular risk factors such as hypertension and dyslipidemia. Significant correlation found between serum resistin levels and age, waist circumference, fasting plasma glucose, lipid profile except for HDL-c levels.

\section{REFERENCES}

1. Ramachandran A, Snehalatha C, Kapur A, Vijay V, Mohan V, Das AK, et al. High prevalence of diabetes and impaired glucose tolerance in India: National Urban Diabetes Survey. Diabetologia. 2001;44(9):1094-101.

2. Dowse GK, Gareeboo H, Zimmet PZ, Alberti KG, Tuomilehto J, Fareed D, et al. High prevalence of NIDDM and impaired glucose tolerance in Indian, Creole, and Chinese Mauritians. Mauritius Noncommunicable Disease Study Group. Diabetes. 1990;39(3):390-6.

3. Bonow RO, Gheorghiade M. The diabetes epidemic: a national and global crisis. The American journal of medicine. 2004;116 Suppl 5A:2S-10S.

4. Janghorbani M, Hu FB, Willett WC, Li TY, Manson JE, Logroscino G, et al. Prospective study of type 1 and type 2 diabetes and risk of stroke subtypes: the Nurses' Health Study. Diabetes care. 2007;30(7):1730-5.

5. Jorgensen H, Nakayama H, Raaschou HO, Olsen TS. Stroke in patients with diabetes. The Copenhagen Stroke Study. Stroke; a journal of cerebral circulation. 1994;25(10):1977-84.

6. Dalal PM. Burden of stroke: Indian perspective. International journal of stroke : official journal of the International Stroke Society. 2006;1(3):164-6.

7. Gorelick PB. Stroke prevention therapy beyond antithrombotics: unifying mechanisms in ischemic stroke pathogenesis and implications for therapy: an invited review. Stroke; a journal of cerebral circulation. 2002;33(3):862-75.

8. Aruna B, Ghosh S, Singh AK, Mande SC, Srinivas V, Chauhan $\mathrm{R}$, et al. Human recombinant resistin protein displays a tendency to aggregate by forming intermolecular disulfide linkages. Biochemistry. 2003;42(36):10554-9.

9. RETN resistin [ Homo sapiens (human) ] Available from: http://www.ncbi.nlm.nih.gov/gene/56729.

10. Gupta V, Singh AK, Kumar S, Srivastava N, Jafar T, Pant AB. Association of circulating resistin with metabolic risk factors in Indian females having metabolic syndrome. Toxicology international. 2011;18(2):168-72.

11. Osawa H, Ochi M, Tabara Y, Kato K, Yamauchi J, Takata Y, et al. Serum resistin is positively correlated with the accumulation of metabolic syndrome factors in type 2 diabetes. Clinical endocrinology. 2008;69(1):7480 .

12. Tangvarasittichai S, Poonsub $\mathrm{P}$, Tangvarasittichai $\mathrm{O}$. Association of serum lipoprotein ratios with insulin resistance in type 2 diabetes mellitus. The Indian journal of medical research. 2010;131:641-8.

\begin{tabular}{|l|}
\hline Source of Support: None Declared \\
Conflict of Interest: None Declared
\end{tabular}

